\title{
Thalamocortical dysconnectivity in schizophrenia
}

Neil D. Woodward, Ph.D., Haleh Karbasforoushan, M.S., and Stephan Heckers, M.D., M.Sc. Psychotic Disorders \& Psychiatric Neuroimaging Programs, Department of Psychiatry, Vanderbilt University School of Medicine, Nashville, TN

\section{Abstract}

Objective-The thalamus and cerebral cortex are connected via topographically organized, reciprocal connections. Previous studies revealed thalamic abnormalities in schizophrenia; however, it is not known if thalamocortical networks are differentially affected in the disorder. To explore this possibility, we examined functional connectivity in intrinsic low frequency bloodoxygen-level-dependent (BOLD) signal fluctuations between major divisions of the cortex and thalamus using resting-state functional magnetic resonance imaging.

Method-77 healthy subjects and 62 patients with schizophrenia underwent resting-state fMRI. To identify functional subdivisions of the thalamus, we parceled the cortex into six regions-ofinterest; prefrontal, motor, somatosensory, temporal, posterior parietal, and occipital cortex. Mean BOLD time-series was extracted from each of the regions-of-interest and entered into a seed-based functional connectivity analysis.

Results-Consistent with prior reports, activity in distinct cortical areas correlated with specific, largely non-overlapping regions of the thalamus in both healthy subjects and schizophrenia patients. Direct comparison between groups revealed reduced prefrontal-thalamic connectivity and increased motor/somatosensory-thalamic connectivity in schizophrenia. The changes in connectivity were unrelated to local grey matter content within the thalamus and antipsychotic medication dosage. No differences were observed in temporal, posterior parietal, and occipital cortex connectivity with the thalamus.

Conclusions-This study establishes differential abnormalities of thalamocortical networks in schizophrenia. The etiology of schizophrenia may disrupt the development of prefrontal-thalamic connectivity and refinement of somatomotor connectivity with the thalamus that occurs during brain maturation.

\section{Keywords}

Schizophrenia; Resting-state fMRI; Functional Connectivity; Thalamus; Cortex

\section{Introduction}

Several neurobiological theories of schizophrenia hypothesize that the pathophysiology of the disorder includes abnormal functional interactions between the cortex and thalamus (1-3). Thalamocortical networks are organized topographically into parallel pathways linking distinct cortical areas to specific thalamic nuclei $(4 ; 5)$. For example, the prefrontal cortex is linked to anterior and dorsomedial areas of the thalamus, whereas motor and

Address Correspondence to: Neil D. Woodward, Ph.D., Assistant Professor of Psychiatry, Psychiatric Neuroimaging \& Psychotic Disorders Programs, Vanderbilt Psychiatric Hospital, Suite 3057, $160123^{\text {rd }}$ Ave. S., Nashville, TN 37212, Phone: 615.322.8361, Fax: 615.936.3563.

Disclosures: No commercial support was received for the preparation of this manuscript and the authors have no conflicts of interest to report. 
somatosensory cortices connect to ventral lateral and ventral posterior-lateral areas of the thalamus, respectively (4). Consequently, dysfunction of thalamocortical networks may provide a parsimonious account for the wide array of clinical and cognitive symptoms observed in schizophrenia (6). Indeed, functional and structural imaging investigations provide broad support for thalamocortical dysfunction (7-12). However, the topographical arrangement of reciprocal connections between cortex and thalamus also raises the distinct possibility that thalamocortical networks may be differentially affected in schizophrenia (13). Unfortunately, determining the anatomical specificity of thalamocortical network dysconnectivity using conventional task-based functional imaging is challenging as it requires using multiple cognitive paradigms that reliability activate distinct cortical areas and their corresponding thalamic targets in healthy subjects as well as patients with schizophrenia. As a result, the anatomical specificity of thalamocortical functional dysconnectivity in schizophrenia remains largely unknown.

Resting-state functional magnetic resonance imaging, which examines correlations in intrinsic low frequency fluctuations in the blood-oxygenation level dependent (BOLD) signal across brain regions, sidesteps many of the limitations associated with conventional task-based functional imaging and has proven exceptionally useful for mapping brain networks. In a series of elegant studies, Zhang et al. systematically mapped functional connectivity between the cortex and thalamus using resting-state fMRI (14;15). Specifically, by parceling the cortex into regions-of-interest that corresponded to prefrontal, motor, somatosensory, temporal, and parietal/occipital cortices, and using them as seeds in a functional connectivity analysis, they were able to show that activity in each cortical area correlated with distinct, largely non-overlapping regions of the thalamus. For instance, prefrontal cortex functionally correlated with anterior and dorsomedial thalamus, whereas motor cortex correlated with ventral lateral thalamus $(14 ; 15)$. Critically, the patterns of intrinsic functional connectivity revealed using resting-state fMRI correspond closely to anatomical connections based on histology and diffusion tensor imaging of white matter pathways (14-18). Consequently, resting-state fMRI may be an ideal method for examining the integrity of functional connectivity between cortex and thalamus in schizophrenia. To this end, we used a similar approach as Zhang et al. $(14 ; 15)$ to examine functional connectivity between cortex and thalamus in schizophrenia and determine if specific thalamocortical networks are differentially affected in the disorder.

\section{Methods}

\section{Participants}

The subjects included in this study were drawn from a dataset of 160 individuals ( 87 healthy subjects and 73 patients with schizophrenia or schizoaffective disorder) with resting-state fMRI data. We refer to the patient group as the schizophrenia group throughout the paper. Patients were recruited through the Vanderbilt Psychotic Disorders Program at the Vanderbilt Psychiatric Hospital in Nashville, Tennessee. This study was approved by the Vanderbilt University Institutional Review Board. All subjects provided written informed consent prior to participating. The Structured Clinical Interview for Diagnosing DSM-IV Disorders (SCID: 19) was administered to confirm diagnoses in patients and rule out current or past psychiatric illness in healthy subjects. Clinical symptoms of psychosis were quantified with the Positive and Negative Syndrome Scale (PANSS: 20). The Wechsler Test of Adult Reading (21) was also administered to provide an estimate of pre-morbid intellect. Exclusion criteria included age less than 16 or greater than 65; estimated pre-morbid IQ less than 70; presence of a systemic medical illness (e.g. diabetes, cardiovascular disease) or central nervous system disorder (e.g. multiple sclerosis, epilepsy) that would affect study results; reported pregnancy or lactation; history of significant head trauma; psychotropic drug use (healthy subjects only); substance abuse within last three months (patients) or 
lifetime history of substance abuse/dependence (healthy subjects); and MRI contraindicators (e.g. metal implants, claustrophobia).

\section{Imaging data acquisition and pre-processing}

Imaging data was collected on one of two identical 3T Philips Intera Achieva MRI scanners, denoted 3T-A and 3T-B, located at Vanderbilt University Institute of Imaging Science. The 7-minute echo-planar imaging resting-state scan had the following parameters: 28 axial slices, matrix $=80 \times 80,3.0 \mathrm{~mm} \times 3.0 \mathrm{~mm}$ in-plane resolution, slice thickness $=4.0 \mathrm{~mm}, 203$ volumes, TR/TE $=2000 / 35 \mathrm{~ms}$. Subjects were instructed to rest quietly with their eyes closed and to remain awake during the scan. In addition, a high resolution T1-weighted fast field echo (FFE) structural scan (170 sagital slices, matrix $=256 \times 256,1.0 \mathrm{~mm}$ isovoxel resolution, $\mathrm{TR} / \mathrm{TE}=8.0 / 3.7 \mathrm{~ms}$ ) was acquired. The resting-state scan was acquired right after the survey and high resolution structural scans, and was not preceded by a cognitive task.

Every subjects' functional imaging data was required to pass our in-house developed quality assurance toolbox that is based on the Biomedical Informatics Research Network Functional Neuroimaging Calibration Study quality assurance protocol (22). Five features (i.e. signalto-noise ratio, percent drift, percent fluctuation, radius of decorrelation, percent standard deviation) were extracted from each scan. Each scan was classified as acceptable if every feature passed pre-determined cut-off points that were derived from a fuzzy clustering algorithm applied to a separate "training" dataset of 84 functional runs obtained from a mixed sample of healthy subjects and patients with a psychotic disorder. Subsequent functional imaging pre-processing was carried out using Statistical Parametric Mapping 8 (http://www.fil.ion.ucl.ac.uk/spm/software/spm8/) and included correction for head motion and slice timing offset, band-pass filtering $(0.01 \mathrm{~Hz}<\mathrm{f}<0.1 \mathrm{~Hz})$, and spatial coregistration to the subject's structural image. Consistent with prior thalamocortical resting-state studies that used the current method, no spatial smoothing was applied to the functional data $(14 ; 15 ; 23)$. Each subject's T1 structural scan was segmented into grey matter, white matter, and cerebrospinal fluid (CSF) tissue classes using the Voxel-Based Morphometry 8 toolbox (http://dbm.neuro.unijena.de/vbm) and the grey matter tissue segment was normalized to the a-priori Montreal Neurological Institute (MNI) grey matter template. Normalization parameters derived from this step were then applied to the functional, white matter, and CSF tissue class images to bring them into MNI space.

\section{Functional connectivity analysis}

As per Zhang et al. (14;15), the cortex was divided into non-overlapping regions-of-interest (prefrontal cortex, motor cortex/supplementary motor area, somatosensory cortex, temporal lobe, posterior parietal cortex, and occipital lobe) which were used as seeds in a seed-tovoxel functional connectivity analysis (see Figure 1A and Supplemental Figure 1). The only deviations from Zhang et al. were that: 1) we used the maximum likelihood maps from the Laboratory of Neuroimaging (LONI) Probabilistic Atlas of cortical structures (24) and Harvard-Oxford supplementary motor area probabilistic atlas (http://www.fmrib.ox.ac.uk/ fsi/) to construct the regions of interest, rather than tracing them on a single subject's brain; and 2) we divided the parietal/occipital region-of-interest into posterior parietal and occipital regions-of-interest. The prefrontal cortex included the superior, middle, and inferior frontal gyri; middle and lateral orbitofrontal gyri; gyrus rectus, and anterior cingulate gyrus. The motor cortex/supplementary motor area region-of-interest included the precentral gyrus and the supplementary motor area. The somatosensory region-of-interest consisted of the postcentral gyrus. The temporal lobe region-of-interest included the superior, middle, and inferior temporal gyri, parahippocampal gyrus, and fusiform gyrus. The posterior parietal region-of-interest included the superior parietal, supramarginal, and angular gyri, posterior cingulate, and precuneus. The occipital region-of-interest included the superior, middle, and 
inferior occipital gyri, lingual gyrus, and cuneus. The cortical regions-of-interest were masked with the LONI probabilistic atlas grey matter tissue map, thresholded at .15, to eliminate voxels with low grey matter intensity.

Functional connectivity maps for each cortical region-of-interest were created for each subject using the CONN-fMRI Functional Connectivity toolbox (http://www.nitrc.org/ projects/conn). Briefly, the mean BOLD time series from each region-of-interest was entered as a predictor in a multiple regression general linear model at each voxel. Regressors corresponding to 6 motion correction parameters and their first temporal derivatives, grey matter, white matter, and CSF were also included to remove variance related to head motion, the global signal, white matter, and CSF, respectively. Regressors for the global, white matter, and CSF signals were created by extracting the BOLD time-courses from the tissue class segmented images (grey matter, white matter, and CSF), averaged across all voxels within each tissue class.

Statistical analysis of functional imaging data proceeded by entering the individual subject functional connectivity maps (in beta-weight units) into random effects analyses to create within group functional connectivity statistical parametric maps and compare connectivity between groups. Only positive correlations were examined. All statistical maps were thresholded at the cluster-level corrected alpha level $\mathrm{p}=.05$ for voxel-wise $\mathrm{p}=.001$, masked to include only voxels within the Harvard-Oxford thalamus atlas.

\section{Results}

10 healthy subjects and 11 patients had resting-state scans that did not pass our quality assurance protocol (see Methods section) and/or had poor quality structural scans (i.e. significant motion artifact). Thus, the final dataset comprised 77 healthy control subjects and 62 schizophrenia patients (schizoaffective disorder $n=24$ ). Subject demographics are presented in Table 1. With the exception of 3 individuals that were not on antipsychotic medication, patients were taking either 1 or 2 atypical antipsychotics $(n=42$ and 9 , respectively), 1 atypical and 1 typical antipsychotic $(n=6)$, or 1 typical antipsychotic $(n=2)$. The groups did not differ on parental education, gender distribution, or ethnicity. As expected, pre-morbid intellect was lower in schizophrenia $(\mathrm{t}(137)=4.84, \mathrm{p}<.001)$. Patients were also slightly older than control subjects ( 36.9 vs. 33.1$)$ at the trend significance level $(\mathrm{t}(137)=1.92, \mathrm{p}=.056)$. Rather than selectively remove younger healthy subjects from the analysis to exactly equate the two groups on age, which would have reduced statistical power and possibly introduced bias, age was included as a covariate in the between group imaging analyses. Chi-square analysis indicated that relatively fewer patients were scanned on the 3T-B scanner (healthy subjects $n=36$, patients $n=16 ; X 2(1)=4.45, p=.011$ ). To ensure that data could be combined across scanners, we compared scanners on the five signal features extracted from the quality assurance protocol (see Methods). None of the features differed between scanners (all $\mathrm{t}(137)<1.71, \mathrm{p}>.091)$.

\section{Thalamocortical Connectivity in Healthy Controls and Patients with Schizophrenia}

Healthy Subjects-As shown in Figure 1B, each cortical region-of-interest was connected to distinct, largely non-overlapping regions of the thalamus. The current results are virtually identical to prior studies using the same method in healthy adults $(14 ; 15 ; 23)$. As expected, the prefrontal cortex was functionally connected to anterior and dorsomedial regions of the thalamus. Motor and somatosensory regions-of-interest correlated strongly with ventral lateral and ventral posterior-lateral portions of the thalamus, respectively. The temporal lobe and occipital cortex correlated with posterior medial and lateral areas of the thalamus that appeared consistent with the medial geniculate nucleus and lateral geniculate 
nucleus, respectively. The posterior parietal cortex was robustly connected with lateral posterior nucleus and pulvinar.

Schizophrenia-As shown in Figure 1C, patients also demonstrated a high degree of segregation in the thalamus and the overall pattern of functional connectivity between cortex and thalamus corresponded well with prior findings in healthy subjects. However, there were qualitative differences between patients and healthy subjects. Specifically, prefrontal cortex connectivity with the thalamus appeared markedly less robust and was restricted largely to the anterior thalamus, whereas the spatial extent of motor and somatosensory connectivity was considerably more extensive in schizophrenia. Specifically, the thalamic clusters showing connectivity with these regions-of-interest were expanded in the dorsal and lateral directions in schizophrenia.

\section{Group Differences in Thalamocortical Connectivity}

Group differences in thalamocortical connectivity are presented in Table 2, and graphically in Figure 1D. Patients demonstrated significantly less connectivity between the prefrontal cortex and bilateral dorsomedial thalamus. In contrast, thalamic connectivity with motor and somatosensory cortex was increased in schizophrenia. No differences in temporal, posterior parietal, and occipital cortex connectivity with the thalamus were observed.

To ensure the group differences in connectivity were not due to antipsychotic medication, structural brain changes in the thalamus, or scanner effects, we extracted the connectivity beta-weights from each cluster identified in the between groups analysis and performed several supplementary analyses. To rule out medication effects, we correlated the cluster beta-weights with current antipsychotic dose, in chlorpromazine (CPZ) equivalents. Functional connectivity did not correlate with CPZ equivalents in any cluster (all Pearson r absolute values $<.17, \mathrm{p}>.18$ ). To rule out the possibility that connectivity differences were due to structural brain differences in the thalamus, we extracted each subject's mean grey matter content within each cluster (i.e. average fraction of grey matter within the cluster) from the grey matter segmented images and performed an ANCOVA analysis on the connectivity beta-weights with mean grey matter content included as a covariate. The group effect was highly significant for each cluster (all p-values<.00005) indicating that the functional connectivity changes observed in schizophrenia were not due to differences in the content of grey matter within each cluster. Finally, to ensure the group effects were not due to differential allocation of patients and healthy subjects to the two scanners, we performed ANCOVA analyses on the beta-weights extracted from the clusters identified in the between groups analysis with scanner included as a covariate. The group effect remained highly significant for all of the clusters (all p-values<.0005). Additionally, the results of the voxelwise analysis with scanner included as a covariate were virtually indistinguishable from the main analysis (see Supplemental Figure 2).

We examined the relationship between the connectivity changes, clinical symptoms (i.e. PANSS positive, negative, and general psychopathology scores), and illness relevant demographics (i.e. duration of illness, pre-morbid IQ). Given the exploratory nature of this analysis, only correlations with alpha levels <.005 were considered significant. No correlations between functional connectivity and clinical symptoms or illness relevant demographics met this threshold.

\section{Discussion}

Dysfunction of thalamocortical networks has been implicated in the pathophysiology of schizophrenia $(2 ; 3)$. We used resting-state fMRI to determine the anatomical specificity of thalamocortical network dysfunction in schizophrenia (15). We found that functional 
connectivity between the prefrontal cortex and dorsomedial/anterior thalamus is reduced in schizophrenia. In contrast, thalamic functional connectivity with motor and somatosensory cortical areas was markedly increased. The results indicate that functional networks linking cortex to thalamus are abnormal in schizophrenia and that the changes are characterized by both hypo- and hyper-connectivity.

The combination of decreased prefrontal-thalamic and increased thalamic connectivity with motor and somatosensory cortical regions is the most striking aspect of our findings which, at first glance, appear inconsistent with the general notion that neural connectivity is overall reduced in schizophrenia (e.g. 25). However, when interpreted from a developmental perspective, the results provide compelling support for the neurodevelopmental model of schizophrenia. Using the same method, Fair and colleagues (23) found marked differences in thalamocortical functional connectivity between children, adolescents, and adults. Specifically, prefrontal-thalamic connectivity is largely absent in children and adolescents suggesting that this network develops abruptly during the transition from adolescence to adulthood. Motor and somatosensory connectivity on the other hand appears to follow an inverted U-curve, being maximal in adolescence compared to childhood and adulthood. The changes observed in schizophrenia may result from abnormal late brain maturation, during the transition from adolescence to adulthood, which derails the normal development of prefrontal-thalamic connectivity and refinement of somatomotor-thalamic connectivity. The functional consequences of these changes remain to be characterized; however, reduced structural connectivity between prefrontal cortex and thalamus has been linked to working memory impairment and prefrontal brain activity in schizophrenia (26). It's possible that a similar relationship may be observed for resting-state functional connectivity.

While the combination of decreased prefrontal and increased somatomotor connectivity with the thalamus is consistent with atypical brain maturation in schizophrenia, the absence of group differences in temporal-thalamic connectivity potentially argues against a neurodevelopmental basis for thalamocortical dysconnectivity. In contrast to prefrontalthalamic connectivity, temporal cortex connectivity with the thalamus decreases with age in typically developing individuals (23). Therefore, patients might be expected to demonstrate increased temporal-thalamic connectivity if thalamocortical network dysfunction is indeed associated with atypical brain maturation. However, the precise timing of developmental changes in thalamocortical functional connectivity is poorly understood. The limited available evidence, which comes from a single cross-sectional study, suggests that much of the reduction in temporal-thalamic connectivity occurs between childhood and adolescence (23). Consequently, it's possible that the developmental disruption in schizophrenia occurs after temporal-thalamic connectivity has fully matured, but before prefrontal and somatomotor thalamic networks have reached adult levels. A better understanding of the normal developmental trajectories of thalamocortical connectivity and investigation of thalamocortical connectivity in first episode schizophrenia is required to test this hypothesis. The lack of group differences in temporal-thalamic, and occipital-thalamic connectivity for that matter, is also interesting given strong evidence of sensory processing deficits in schizophrenia (27). It's possible that connectivity during tasks, rather than resting-state, may be associated with sensory processing dysfunction. Alternatively, auditory and visual sensory processing deficits in schizophrenia may relate to dysfunction at the level of corticocortical interactions, rather thalamocortical connectivity.

Our findings also raise the possibility that thalamocortical dysconnectivity results from selective pathology of one or more nuclei of the thalamus and/or their corresponding cortical targets. Reduced number of neurons in the mediodorsal thalamus has been found by several investigators; although there are also reports of normal numbers of neurons (see 13 for review). Similarly, an array of neuronal and molecular changes have also been found in 
dorsolateral prefrontal cortex circuitry (see 28 for review). As such, thalamocortical dysconnectivity in schizophrenia may result from selective pathology of specific thalamic nuclei, and/or their corresponding cortical targets. Resting-state connectivity networks are conserved across species suggesting that animal models will be particularly useful in elucidating the effects of focal neuronal, molecular, and genetic manipulations on largescale brain networks (29-33).

The current results are also informed by considering the physiology of BOLD functional connectivity. There is considerable overlap between functional and structural connectivity in the thalamus suggesting that thalamocortical functional connectivity reflects direct anatomical connections (14). Results from a recent diffusion tensor imaging investigation showing reduced connectivity between thalamus and lateral prefrontal cortex, and increased connectivity between somatosensory cortex and thalamus in schizophrenia provide a potential anatomical basis for the current findings (26). However, it is clear from the broader functional connectivity literature that brain regions not directly anatomically connected can still demonstrate robust functional connectivity indicating that resting-state connectivity networks reflect extended, polysynaptic networks (e.g. 34). This interpretation is supported by findings from a recent combined resting-state fMRI/electrocorticography investigation which found that functional connectivity, positive correlations in particular, predicted electrically evoked brain responses (35). Combined, these findings confirm a neural basis for low frequency BOLD functional connectivity, but raise the possibility that abnormal thalamocortical connectivity in schizophrenia may reflect alterations in direct and/or indirect pathways linking thalamus and cortex. Future work combining functional and structural connectivity will help clarify the nature of thalamocortical dysconnectivity in schizophrenia.

There are several limitations of the current investigation that merit consideration. First, patients were receiving antipsychotic medication. While we did not find any evidence that medication was related to functional connectivity abnormalities observed in patients; it's possible that antipsychotic treatment effects on connectivity may not be dose-dependent. Second, low frequency BOLD functional connectivity varies to some extent across cognitive states, levels of consciousness (i.e. awake vs. light sleep), and even eyes open vs. closed conditions (36-39). Since we instructed subjects to keep their eyes closed during scanning, we can not rule out the possibility that some subjects may have fallen asleep during scanning and that group differences in arousal may have contributed to the results. Studies examining thalamocortical connectivity across cognitive states and levels of arousal will be required to determine if the alterations observed in patients transcend cognitive state and arousal level. Moreover, resting-state connectivity is modified by recent experiences raising the possibility that the abnormalities in thalamocortical connectivity are secondary to negative life experiences associated with illness chronicity, such as long-term reduction in social interaction and cognitive engagement, rather than the pathophysiology of schizophrenia (40). Third, while we argue that the changes are at least partially consistent with neurodevelopmental hypotheses of schizophrenia, it is premature to exclude a neurodegenerative explanation for the findings. A combination of decreased network connectivity with compensatory increases in other networks has been observed in degenerative illnesses such as Alzheimer's disease (41). Replication of the current findings in unmedicated/minimally treated first-episode or early-phase patients will strengthen the case for a neurodevelopmental basis for thalamocortical dysconnectivity. Finally, although we applied a well-established method to examine thalamocortical functional connectivity, there are nonetheless limitations of the technique. The use of large cortical areas as seeds, while useful for functionally segregating the thalamus, does not allow for a more finegrained analysis at the cortical level. Interestingly, using a voxel located in the mediodorsal thalamus as the seed for functional connectivity analysis, Welsh and colleagues found that mediodorsal thalamic connectivity with bilateral caudate and anterior cingulate gyrus was 
reduced in schizophrenia (12). Follow-up investigation using the thalamic clusters identified in the current study as seeds in an independent cohort of patients, or independent components analysis, may help further refine the anatomical specificity of thalamocortical dysconnectivity.

In conclusion, we found altered resting-state functional connectivity between the thalamus and cortex is altered in schizophrenia. The alterations are characterized by decreased prefrontal-thalamic connectivity, and increased thalamic connectivity with motor and somatomotor cortex. Combined, the results implicate abnormal late brain maturation in the neuropathology of schizophrenia. Future studies combining functional connectivity with assessment of phenotypes more closely related to thalamocortical networks than complex, clinical symptoms may help elucidate the functional consequences of thalamocortical dysconnectivity in schizophrenia.

\section{Supplementary Material}

Refer to Web version on PubMed Central for supplementary material.

\section{Acknowledgments}

This research was supported by funding from the NIMH (RO1-MH070560 awarded to SH), the Vanderbilt Psychiatric Genotype/Phenotype Project, the Brain and Behavior Research Fund (NARSAD Young Investigator Award awarded to NDW), and the Vanderbilt Institute for Clinical and Translational Research (1-UL1-RR024975 NCRR/NIH). The authors are indebted to the individuals who participated in the study. We thank Kristan Armstrong, Julia Sheffield and Austin Woolard for their assistance recruiting and screening subjects for participation in the study.

\section{Reference List}

1. Swerdlow NR. Integrative circuit models and their implications for the pathophysiologies and treatments of the schizophrenias. Curr Top Behav Neurosci. 2010; 4:555-583. [PubMed: 21312413]

2. Andreasen NC, O'Leary DS, Cizadlo T, Arndt S, Rezai K, Ponto LL, Watkins GL, Hichwa RD. Schizophrenia and cognitive dysmetria: a positron-emission tomography study of dysfunctional prefrontal-thalamic-cerebellar circuitry. Proc Natl Acad Sci U S A. Sep 3; 1996 93(18):9985-9990. [PubMed: 8790444]

3. Jones EG. Cortical development and thalamic pathology in schizophrenia. Schizophr Bull. 1997; 23(3):483-501. [PubMed: 9327511]

4. Alexander GE, DeLong MR, Strick PL. Parallel organization of functionally segregated circuits linking basal ganglia and cortex. Annu Rev Neurosci. 1986; 9:357-381. [PubMed: 3085570]

5. Haber SN. The primate basal ganglia: parallel and integrative networks. J Chem Neuroanat. 2003; 26(4):317-330. [PubMed: 14729134]

6. Andreasen NC, Paradiso S, O'Leary DS. "Cognitive dysmetria" as an integrative theory of schizophrenia: a dysfunction in cortical-subcortical-cerebellar circuitry? Schizophr Bull. 1998; 24(2):203-218. [PubMed: 9613621]

7. Shenton ME, Dickey CC, Frumin M, McCarley RW. A review of MRI findings in schizophrenia. Schizophr Res. Apr 15; 2001 49(1-2):1-52. [PubMed: 11343862]

8. Glahn DC, Laird AR, Ellison-Wright I, Thelen SM, Robinson JL, Lancaster JL, Bullmore E, Fox PT. Meta-analysis of gray matter anomalies in schizophrenia: application of anatomic likelihood estimation and network analysis. Biol Psychiatry. Nov 1; 2008 64(9):774-781. [PubMed: 18486104]

9. Andreasen NC. The role of the thalamus in schizophrenia. Can J Psychiatry. 1997; 42(1):27-33. [PubMed: 9040920]

10. Minzenberg MJ, Laird AR, Thelen S, Carter CS, Glahn DC. Meta-analysis of 41 functional neuroimaging studies of executive function in schizophrenia. Arch Gen Psychiatry. 2009; 66(8): 811-822. [PubMed: 19652121] 
11. Andreasen NC, Pierson R. The role of the cerebellum in schizophrenia. Biol Psychiatry. Jul 15; 2008 64(2):81-88. [PubMed: 18395701]

12. Welsh RC, Chen AC, Taylor SF. Low-frequency BOLD fluctuations demonstrate altered thalamocortical connectivity in schizophrenia. Schizophr Bull. 2010; 36(4):713-722. [PubMed: 18990709]

13. Sim K, Cullen T, Ongur D, Heckers S. Testing models of thalamic dysfunction in schizophrenia using neuroimaging. J Neural Transm. 2006; 113(7):907-928. [PubMed: 16252070]

14. Zhang D, Snyder AZ, Shimony JS, Fox MD, Raichle ME. Noninvasive functional and structural connectivity mapping of the human thalamocortical system. Cereb Cortex. 2010; 20(5):11871194. [PubMed: 19729393]

15. Zhang D, Snyder AZ, Fox MD, Sansbury MW, Shimony JS, Raichle ME. Intrinsic functional relations between human cerebral cortex and thalamus. J Neurophysiol. 2008; 100(4):1740-1748. [PubMed: 18701759]

16. Behrens TE, Johansen-Berg H, Woolrich MW, Smith SM, Wheeler-Kingshott CA, Boulby PA, Barker GJ, Sillery EL, Sheehan K, Ciccarelli O, Thompson AJ, Brady JM, Matthews PM. Noninvasive mapping of connections between human thalamus and cortex using diffusion imaging. Nat Neurosci. 2003; 6(7):750-757. [PubMed: 12808459]

17. Johansen-Berg H, Behrens TE, Sillery E, Ciccarelli O, Thompson AJ, Smith SM, Matthews PM. Functional-anatomical validation and individual variation of diffusion tractography-based segmentation of the human thalamus. Cereb Cortex. 2005; 15(1):31-39. [PubMed: 15238447]

18. Klein JC, Rushworth MF, Behrens TE, Mackay CE, de Crespigny AJ, D’Arceuil H, Johansen-Berg $\mathrm{H}$. Topography of connections between human prefrontal cortex and mediodorsal thalamus studied with diffusion tractography. Neuroimage. 2010; 51(2):555-564. [PubMed: 20206702]

19. First, MB.; Spitzer, RL.; Gibbon, M.; Williams, JBW. Structured Clinical Interview for DSM-IV Axis I Disorders. Clinical Version (SCID-CV). Washington, D.C.: American Psychiatric Press Inc; 1996.

20. Kay SR, Fiszbein A, Opler LA. The positive and negative syndrome scale (PANSS) for schizophrenia. Schizophr Bull. 1987; 13(2):261-276. [PubMed: 3616518]

21. Wechsler, D. Wechsler Test of Adult Reading. San Antonio, TX: Pearson; 2001.

22. Friedman L, Glover GH. Report on a multicenter fMRI quality assurance protocol. J Magn Reson Imaging. 2006; 23(6):827-839. [PubMed: 16649196]

23. Fair DA, Bathula D, Mills KL, Dias TG, Blythe MS, Zhang D, Snyder AZ, Raichle ME, Stevens AA, Nigg JT, Nagel BJ. Maturing thalamocortical functional connectivity across development. Front Syst Neurosci. 2010; 4:10. [PubMed: 20514143]

24. Shattuck DW, Mirza M, Adisetiyo V, Hojatkashani C, Salamon G, Narr KL, Poldrack RA, Bilder RM, Toga AW. Construction of a 3D probabilistic atlas of human cortical structures. Neuroimage. Feb 1; 2008 39(3):1064-1080. [PubMed: 18037310]

25. Karlsgodt KH, Sun D, Jimenez AM, Lutkenhoff ES, Willhite R, van Erp TG, Cannon TD. Developmental disruptions in neural connectivity in the pathophysiology of schizophrenia. Dev Psychopathol. 2008; 20(4):1297-1327. [PubMed: 18838043]

26. Marenco S, Stein JL, Savostyanova AA, Sambataro F, Tan HY, Goldman AL, Verchinski BA, Barnett AS, Dickinson D, Apud JA, Callicott JH, Meyer-Lindenberg A, Weinberger DR. Investigation of Anatomical Thalamo-Cortical Connectivity and fMRI Activation in Schizophrenia. Neuropsychopharmacology. Sep 28.2011

27. Leitman DI, Sehatpour P, Higgins BA, Foxe JJ, Silipo G, Javitt DC. Sensory deficits and distributed hierarchical dysfunction in schizophrenia. Am J Psychiatry. 2010; 167(7):818-827. [PubMed: 20478875]

28. Volk DW, Lewis DA. Prefrontal cortical circuits in schizophrenia. Curr Top Behav Neurosci. 2010; 4:485-508. [PubMed: 21312410]

29. Vincent JL, Patel GH, Fox MD, Snyder AZ, Baker JT, Van Essen DC, Zempel JM, Snyder LH, Corbetta M, Raichle ME. Intrinsic functional architecture in the anaesthetized monkey brain. Nature. May 3; 2007 447(7140):83-86. [PubMed: 17476267] 
30. Margulies DS, Vincent JL, Kelly C, Lohmann G, Uddin LQ, Biswal BB, Villringer A, Castellanos FX, Milham MP, Petrides M. Precuneus shares intrinsic functional architecture in humans and monkeys. Proc Natl Acad Sci U S A. Nov 24; 2009 106(47):20069-20074. [PubMed: 19903877]

31. Liang Z, King J, Zhang N. Uncovering intrinsic connectional architecture of functional networks in awake rat brain. J Neurosci. Mar 9; 2011 31(10):3776-3783. [PubMed: 21389232]

32. Magnuson M, Majeed W, Keilholz SD. Functional connectivity in blood oxygenation leveldependent and cerebral blood volume-weighted resting state functional magnetic resonance imaging in the rat brain. J Magn Reson Imaging. 2010; 32(3):584-592. [PubMed: 20815055]

33. Zhang N, Rane P, Huang W, Liang Z, Kennedy D, Frazier JA, King J. Mapping resting-state brain networks in conscious animals. J Neurosci Methods. Jun 15; 2010 189(2):186-196. [PubMed: 20382183]

34. Honey CJ, Sporns O, Cammoun L, Gigandet X, Thiran JP, Meuli R, Hagmann P. Predicting human resting-state functional connectivity from structural connectivity. Proc Natl Acad Sci U S A. Feb 10; 2009 106(6):2035-2040. [PubMed: 19188601]

35. Keller CJ, Bickel S, Entz L, Ulbert I, Milham MP, Kelly C, Mehta AD. Intrinsic functional architecture predicts electrically evoked responses in the human brain. Proc Natl Acad Sci U S A. Jun 21; 2011 108(25):10308-10313. [PubMed: 21636787]

36. Van Dijk KR, Hedden T, Venkataraman A, Evans KC, Lazar SW, Buckner RL. Intrinsic functional connectivity as a tool for human connectomics: theory, properties, and optimization. $\mathrm{J}$ Neurophysiol. 2010; 103(1):297-321. [PubMed: 19889849]

37. Larson-Prior LJ, Zempel JM, Nolan TS, Prior FW, Snyder AZ, Raichle ME. Cortical network functional connectivity in the descent to sleep. Proc Natl Acad Sci U S A. Mar 17; 2009 106(11): 4489-4494. [PubMed: 19255447]

38. Hampson M, Olson IR, Leung HC, Skudlarski P, Gore JC. Changes in functional connectivity of human MT/V5 with visual motion input. Neuroreport. Jun 7; 2004 15(8):1315-1319. [PubMed: 15167557]

39. Hampson M, Driesen NR, Skudlarski P, Gore JC, Constable RT. Brain connectivity related to working memory performance. J Neurosci. Dec 20; 2006 26(51):13338-13343. [PubMed: 17182784]

40. Stevens WD, Buckner RL, Schacter DL. Correlated low-frequency BOLD fluctuations in the resting human brain are modulated by recent experience in category-preferential visual regions. Cereb Cortex. 2010; 20(8):1997-2006. [PubMed: 20026486]

41. Zhou J, Greicius MD, Gennatas ED, Growdon ME, Jang JY, Rabinovici GD, Kramer JH, Weiner M, Miller BL, Seeley WW. Divergent network connectivity changes in behavioural variant frontotemporal dementia and Alzheimer's disease. Brain. 2010; 133(Pt 5):1352-1367. [PubMed: 20410145] 


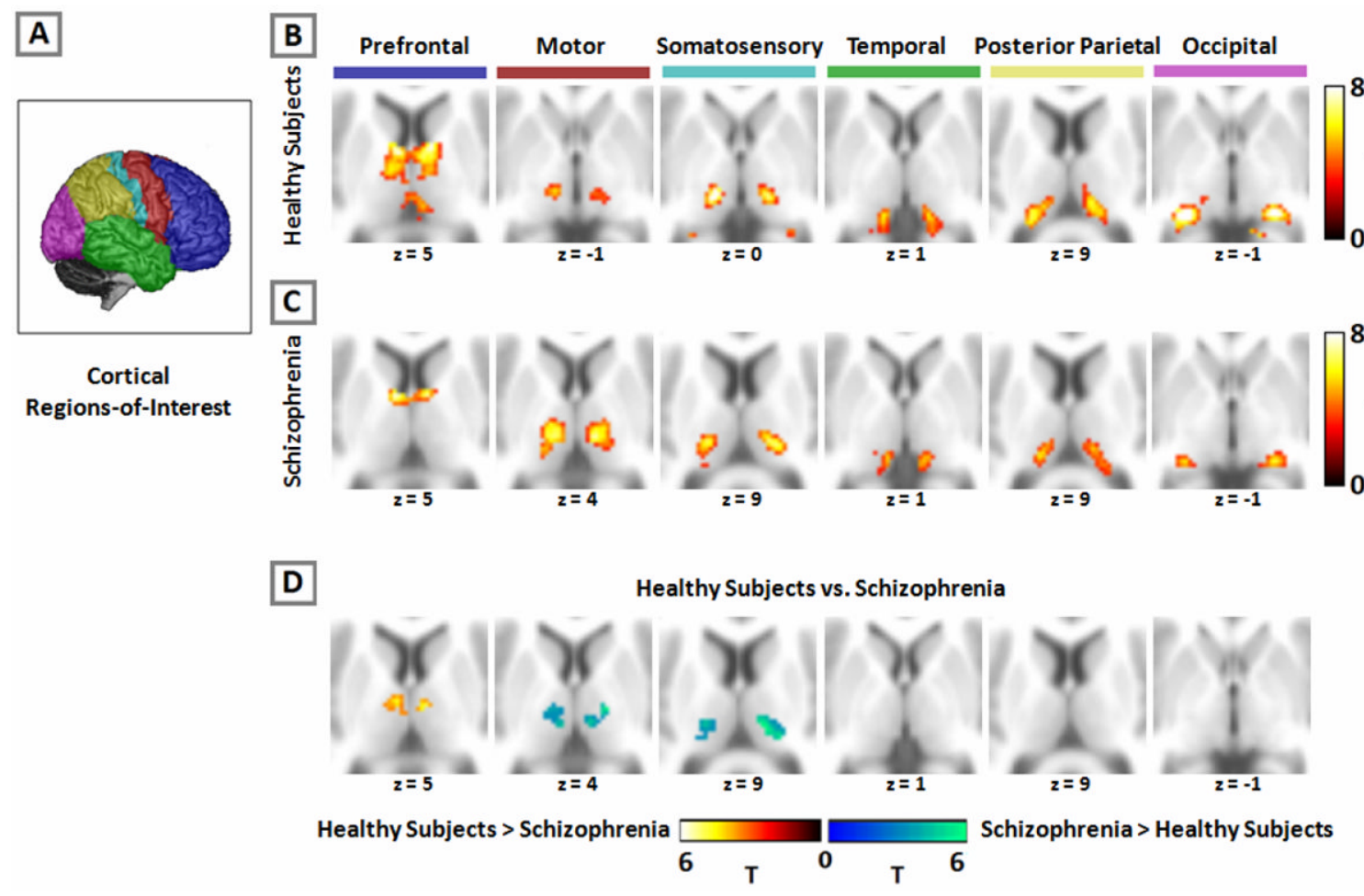

Figure 1.

Resting-state functional connectivity between cortex and thalamus is altered in schizophrenia. The cortex is partitioned into six, non-overlapping regions-of-interest which were used as seeds in a functional connectivity analysis (panel A). Activity in each cortical region-of-interest correlated with distinct areas of the thalamus in both healthy subjects (panel B) and patients with schizophrenia (panel C). Comparison between groups revealed decreased prefrontal connectivity with the thalamus, and increased motor and somatosensory thalamic connectivity in schizophrenia (panel D). Images thresholded at $\mathrm{p}=.05$ (cluster-wise corrected) for voxel-wise $\mathrm{p}=.001$. We used a similar presentation format as Zhang et al. $(14,15)$ and Fair et al. $(23)$ to facilitate comparison of the current results with prior findings in healthy subjects. 


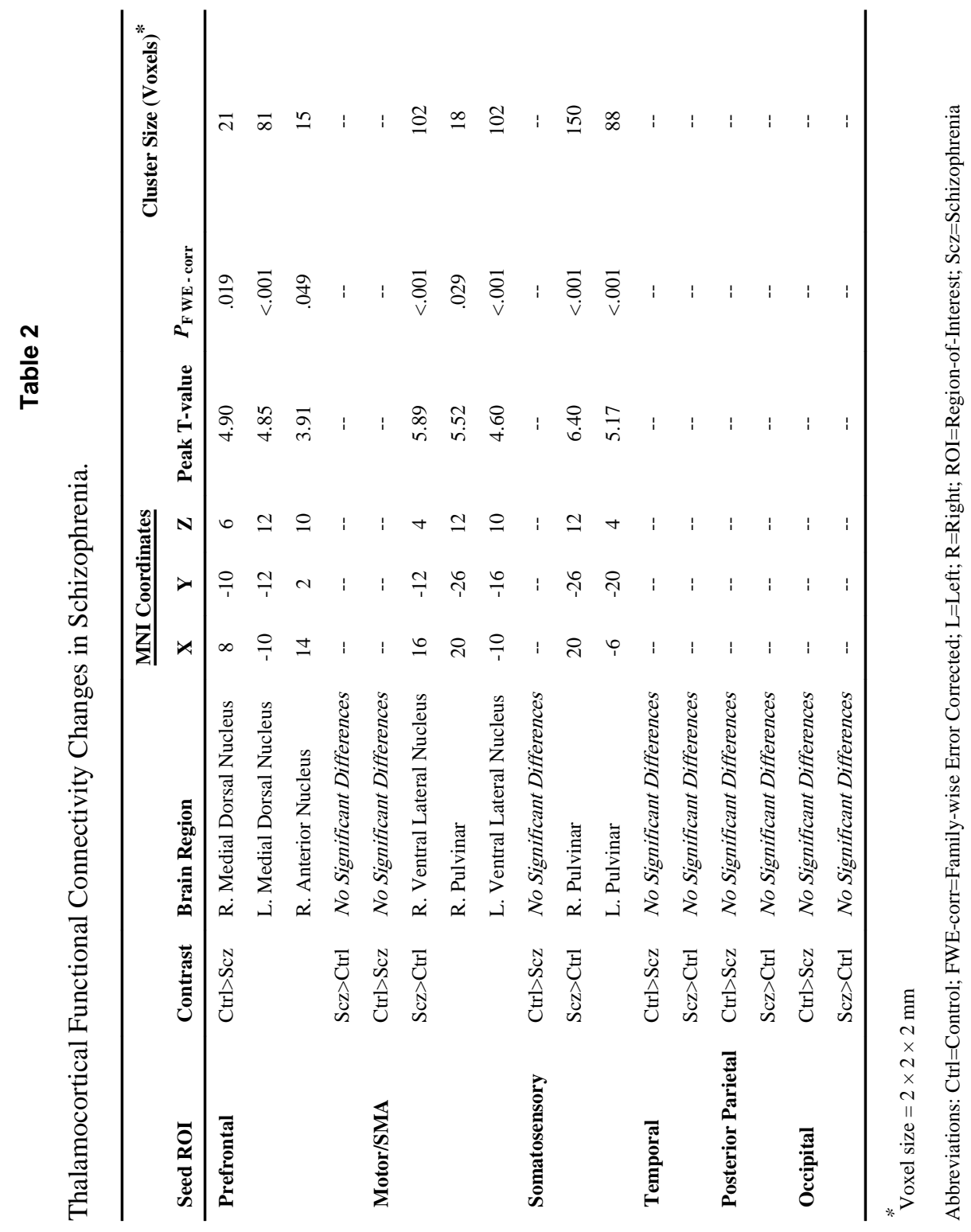

